\title{
The Medical Student Response to the Mental Health Consequences of COVID-19
}

\author{
Anthony N. Almazan ${ }^{1}$ (D) $\cdot$ Andrew S. Chun ${ }^{1} \cdot$ India Perez-Urbano ${ }^{2}$ \\ Received: 13 May 2020 / Revised: 11 August 2020 / Accepted: 8 September 2020 / Published online: 21 September 2020 \\ (C) Academic Psychiatry 2020
}

To the Editor:

As the worldwide number of coronavirus disease 2019 (COVID-19) cases continues to rise, the medical community has identified another imminent crisis - the mental health consequences of the pandemic. An estimated $45 \%$ of American adults feel that COVID-19 has had a negative impact on their mental health [1]. As physical distancing measures remain in place, medical providers continue to anticipate surges in isolation-related mental health conditions, including depression, anxiety, and suicide. Shelter-in-place orders may lead to increasing rates of substance use relapse, overdose, domestic violence, and child abuse [2]. Furthermore, the trauma of caring for individuals with COVID-19, felt by both frontline health care providers and the general public, will likely have lasting consequences. The threat of an enduring mental health crisis has led physicians to call for early preventive efforts [2]. However, the increased burden of mental illness resulting from this pandemic will likely place additional strain on an already overextended mental health care system. The medical community must leverage all of its available assets to combat this threat. We believe that medical students represent a significant untapped resource that should be mobilized to address this impending mental health crisis.

At the time of the writing of this manuscript, the Association of American Medical Colleges stated, "Unless there is a critical health care workforce need locally, we strongly suggest that medical students not be involved in any direct patient care activities" [3]. At the height of the pandemic, medical schools across the country subsequently shifted to virtual learning and barred students from in-person

Anthony N. Almazan

anthony_almazan@hms.harvard.edu

1 Harvard Medical School, 25 Shattuck Street, Boston, MA 02115, USA

2 University of California-San Francisco, San Francisco, CA, USA clinical work. In response, students developed new roles to serve their communities. They made headlines collecting personal protective equipment for hospitals, providing child care for essential workers, and ensuring food security for high-risk community members [4]. Medical students also advocated for their incorporation into telemedicine efforts in order to relieve overburdened medical providers [5]. Missing from this discourse was a discussion of the ways medical students could be voluntarily mobilized to address the mental health challenges resulting from this pandemic.

The resistance to medical student involvement in the COVID-19 response is based on reasonable safety concerns, but a carefully designed medical student mental health response could avert these risks. Recently proposed interventions to address the mental health consequences of COVID19 were designed for remote delivery [2]. The use of digital teleconferencing programs to deliver these interventions would enable medical students to avoid exacerbating infection risks and shortages of personal protective equipment. Whereas medical students may present a liability in the direct care of patients with COVID-19, they are well suited to provide remote mental health support. We propose the following three interventions as examples of a medical student response to the mental health consequences of COVID-19.

First, medical students could serve in a preventive mental health system operating by a stepped care model. Under the supervision of primary care physicians, students could use telehealth systems to perform thorough mental wellness checks across large patient panels. This cannot currently be offered by physicians due to patient care burdens related to COVID-19. Students could fill these gaps by performing standardized mental health screens, offering psychoeducation, and alerting providers about patients who require a higher level of psychiatric care. Such a system could be crucial for identifying isolation-related experiences including domestic violence, child abuse, and substance use relapse, which may go unnoticed as resources are diverted to the COVID-19 response. This triage strategy would alleviate the burden on health care 
providers while bolstering the system's ability to identify cases of mental illness and violence.

Second, medical students could create virtual spaces that minimize the impact of social isolation. Through their clinical rotations, many students obtain experience leading therapeutic support groups intended to foster community and aid patients in managing their illness. As physical distancing measures continue, students could lead virtual spaces for community members, frontline health care workers, and family members of those with COVID-19 to process their experiences, learn evidence-based coping skills, and connect with others. A "see one, do one, teach one" approach has been used by the authors' institutions to prepare students to facilitate mental health support groups, and this strategy could also be used to train and supervise students leading virtual community spaces. This intervention would actively normalize community-based discussions about mental illness while facilitating the crucial reorientation of our communities toward a greater openness about mental health.

Third, medical students could be trained to staff existing mental health hotlines to assist the first responders of this growing mental health crisis. Through their early clinical experiences, medical students hone their communication skills and refine their abilities to support patients in times of distress. These experiences make medical students uniquely positioned to contribute to crisis lines like the National Suicide Prevention Lifeline. Since the beginning of the COVID-19 pandemic, these hotlines have been experiencing significant surges in call volumes. Students could be mobilized to serve through these organizations as sources of support for populations who are not currently connected to the mental health care system.

Medical students cannot answer this call to service without institutional action. These interventions will require physicians to imagine new strategies for task-shifting, invest in the counseling skills of trainees, and develop systems for supervising these student-driven programs. Furthermore, the creation of sustainable student-driven mental health programs will require institutional changes that enable students to dedicate themselves to this cause. For example, granting degree credit to students participating in the aforementioned interventions would facilitate student contributions to the COVID-19 response. If we hope to meaningfully address the possibility of a mental health crisis, medical institutions must formally recognize their students as valuable partners in this historic moment.

In conclusion, while physicians prepare plans to minimize the mental health impact of COVID-19, they will need to develop new strategies to address increasing burdens of mental illness in the context of existing workforce shortages. As patients continue to feel the weight of COVID-19, medical students should be mobilized to respond to the mental health consequences of this pandemic.

Acknowledgments The authors would like to thank Katherine Shaffer and Mimi Yen-Li for their comments on preliminary drafts of this article.

\section{Compliance with ethical standards}

Disclosures On behalf of all authors, the corresponding author states that there is no conflict of interest.

\section{References}

1. KFF Health Tracking Poll - Early April 2020: the impact of coronavirus on life in America. The Henry J Kaiser Family Foundation. April 2, 2020. Available at: https://www.kff.org/health-reform/ report/kff-health-tracking-poll-early-april-2020/. Accessed 1 May 2020.

2. Galea S, Merchant RM, Lurie N. The mental health consequences of COVID-19 and physical distancing: the need for prevention and early intervention. JAMA Intern Med. 2020;180:817-8. https://doi. org/10.1001/jamainternmed.2020.1562.

3. Association of American Medical Colleges. Guidance on Medical Students' Participation in Direct Patient Contact Activities. April 14, 2020. Available at: https://www.aamc.org/system/files/2020-04/ meded-April-14-Guidance-on-Medical-Students-Participation-inDirect-Patient-Contact-Activities.pdf. Accessed 13 May 2020.

4. Krieger P, Goodnough A. Medical students, sidelined for now, find new ways to fight coronavirus. The New York Times. March 23, 2020. Available at: https://www.nytimes.com/2020/03/23/health/ medical-students-coronavirus.html. Accessed 10 Apr 2020.

5. Miller DG, Pierson L, Doernberg S. The role of medical students during the COVID-19 pandemic. Ann Intern Med. 2020;173:145-6. https://doi.org/10.7326/M20-1281.

Publisher's Note Springer Nature remains neutral with regard to jurisdictional claims in published maps and institutional affiliations. 